\title{
Do Intracoronary Techniques Add Diagnostic Value Beyond Angiography in the Characterization of Myocardial Bridges?
}

\author{
Pablo Ramos-Ruiz ${ }^{1 *}$, Hernán David Mejía-Rentería ${ }^{2}$, José Domingo Cascón-Pérez ${ }^{1}$ and Luciano Consuegra- \\ Sánchez ${ }^{1}$
}

${ }^{1}$ Cardiac Hemodynamics Unit, Santa Lucia University Hospital, Spain

${ }^{2}$ Cardiac Hemodynamics Unit, San Carlos Clinical University Hospital, Spain

*Corresponding author: Pablo Ramos Ruiz, Cardiac Hemodynamics Unit, Cardiology

Service, University Hospital of Santa Lucia, Cartagena, Spain.

Received Date: July 22, 2019

Published Date: August 02, 2019
Case Report

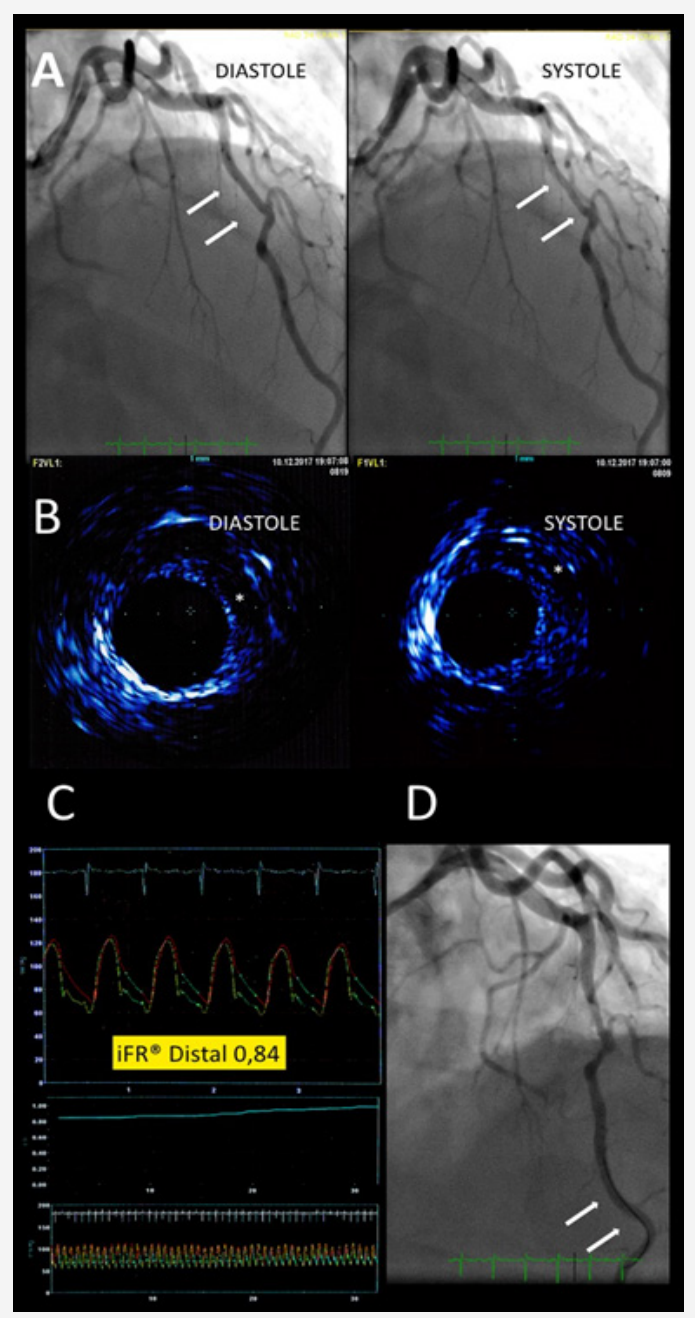

Figure 1: The coronary angiography.
Myocardial bridging (MB) is defined as myocardial tissue surrounding a segment of an epicardial coronary artery that goes intramurally, mostly involving the left anterior descending artery (LAD). Although patients with MB are often asymptomatic, this anomaly has been related to a wide spectrum of symptoms [1]. Pathogenic mechanisms leading to ischemia in MBs are not completely ascertained [2]. In this regard, angiography alone is often inadequate to fully appraise morphology and funcional repercussion of a MB. Recently, the instantaneous wave-free ratio (iFR), a resting trans- stenotic pressure gradient measured at the diastolic "wavefree" period, has been proposed as a promising tool in assessing the hemodynamic relevance of MBs [1,3]. A 59-year-old male with hypertension and dyslipidemia was referred to our department due to stable effort angina and a stress echocardiography showing reversible ischemia in the LAD myocardial territory. The coronary angiography Figure 1, Panel A showed a tunneled segment of the mid LAD with systolic compression, "milking effect" (white arrows). Given the clinical presentation, a comprehensive imaging and functional intracoronary study was performed in order to better characterize the LAD-MB. The intravascular ultrasound (IVUS) study Figure 1, Panel B demostraste a systolic concentric compression of the bridged segment, that persists into diastole with the typical finding of the "half- moon phenomenon", an echolucent area present between the bridged coronary segment and epicardial tissue (white asterisk) [1]. The cross-sectional lumen area variation was $22 \%$. Of note, IVUS ruled-out significant atherosclerotic burden, coronary dissection and other complications associated with MB. The functional assessment of the mid LAD-MB revealed a significant resting diastolic pressure gradient, as measured by iFR (iFR 0.84, Figure 1, Panel C) with the sensor of the pressure-wire 
located distal to MB segment (whit arrows, Figure 1, Panel D). Given the demonstrated functional repercussion of the $\mathrm{MB}$, concordante with the results of the stress echocardiogram and the patient symptoms, it was decided to optimize the medical treatment aimed to the control of angina. However, three months later, our patient persists symptomatic. Here, in summary, we present a case in which imaging and intracoronary physiology techniques were useful for a better morphological and functional assessment of a MB.

\section{Acknowledgement}

None.

\section{Conflict of Interest}

The authors declare no conflict of interest.

\section{References}

1. Tarantini G, Migliore F, Cademartiri F, Fraccaro C, Iliceto S, et al. (2016) The Present and Future Left Anterior Descending Artery Myocardial Bridging A Clinical Approach. J Am Coll Cardiol 68: 2887-2899.

2. Gould KL, Johnson NP (2015) Myocardial Bridges: Lessons in Clinical Coronary Pathophysiology. JACC Cardiovasc Imaging 8(6): 705-709.

3. Gili S, Taha S, Omedé P, Moretti C (2015) A bridge over troubled water: Functional assessment of a myocardial bridge. Int J Cardiol 201: 285287. 